\title{
The comparative accuracy of photographic observations of radio stars observed at the Engelhardt Astronomical Observatory ${ }^{\star}$
}

\author{
N. Rizvanov, I. Dautov, and R. Shaimukhametov \\ Engelhardt Astronomical Observatory, Kazan University, Zelenodolsky region, 422526 Tatarstan, Russia \\ Received 20 December 2000 / Accepted 11 April 2001

\begin{abstract}
At the Engelhardt Astronomical Observatory (EAO), we observed photographic positions of 113 Galactic Radio Sources (GRS) in the system PPM catalogue (Rizvanov \& Dautov 1998). Analysis of their accuracy is made by comparison with the Hipparcos catalogue (Perryman et al. 1997) and astrometric catalogue of radio stars in the radio window from the article of Walter et al. (1991).
\end{abstract}

Key words. astrometry - radio continuum: stars

\section{Introduction}

The IAU in its XXIII General Assembly adopted a new conventional celestial reference system - International Celestial Reference System (ICRS) and a radio frame based on observations of several hundred Extragalactic Radio Sources (ERS) by methods of VLBI since January 1, 1998. The catalogues Hipparcos and Tycho, which are based on data from the space observatory Hipparcos, represent the ICRS in the optical wavelenghts (Perryman et al. 1997). Thus, there are three celestial systems of coordinates at present: the ground-based optical - FK5, the radioastrometrical - ICRS (Ma \& Feissel 1997) and the space optical - Hipparcos Reference Frame (Perryman et al. 1997). By the authors definition, "the ICRS, as represented by the Hipparcos and Tycho Catalogues, replaces the FK5 system as the practical definition of celestial coordinates in the optical region" (Perryman et al. 1997).

Each of these three systems of coordinates was constructed using very different methods. They form internally consistent systems which differ from each other in some. One of the main problems of modern astrometry is to refine connection between these three systems of coordinates. To solve this problem Extragalactic Radio Sources and Galactic Radio Sources (GRS) would be appropriate for use. However, GRS have large proper motions. Therefore they require reobservation.

\footnotetext{
Send offprint requests to: N. Rizvanov, e-mail: Naufal.Rizvanov@ksu.ru

* Table 2 is only available in electronic form at the CDS via anonymous ftp to cdsarc.u-strasbg.fr (130.79.128.5) or via

http://cdsweb.u-strasbg.fr/cgi-bin/qcat?J/A+A/375/670
}

\section{Determination of the coordinates of the Galactic radio sources from the photographic observations of the Zelenchuk mountain station of EAO}

Walter et al. (1991) give the list of 200 GRS in the limits from $6^{\mathrm{m}}$ to $10^{\mathrm{m}}$ as the control points for establishing, connection between, a three systems of coordinates. However, we used the list of the radio sources made by of Svidunovich (private communication). This list contains 205 GRS of from $2^{\mathrm{m}}$ to $16^{\mathrm{m}}$ distributed throughout the celestial sphere. 113 objects were chosen for this study.

122 plates with GRS were obtained by the wide-field astrograph Zeiss $(f=2000 \mathrm{~mm}, D=400 \mathrm{~mm})$ of the Zelenchuk mountain station of EAO in 1992-1994. The plates of the northern and southern sky observed by this telescope in 1984-1989 under the programs FON (Kolchynsky \& Onegina 1979) and FOCAT (Polojntsev \& Potter 1979) were also used to determine the coordinates of radio sources.

We will not describe the method of reduction of astrophotographies of GRS in of detail. We will only note that the process of reduction was automated as much as possible. $15-25$ reference stars of the PPM catalogue were chosen on each plate. The measurements were made by the measuring machine "Ascorecord". While reducing the plates, the influence of the radial cubic distortion relative to the centre of its symmetry (Rizvanov \& Kitkin 1993) was excluded from the measured coordinates. The differential refraction (Rizvanov \& Dautov 1998) relative to the optical centres of the plates was entered into the standard coordinates of the reference stars. Reduction of the 
Table 1. Mean differences in $\alpha$ and $\delta$ between GRS and Hipparcos, Walter (in radio window) catalogues.

\begin{tabular}{lrcrcc}
\hline Catalogue & $\Delta \alpha \cos \delta^{\prime \prime}$ & $\sigma_{\alpha}^{\prime \prime}$ & $\Delta \delta^{\prime \prime}$ & $\sigma_{\delta}^{\prime \prime}$ & $\begin{array}{c}\text { Common } \\
\text { star }\end{array}$ \\
\hline Hipparcos & 0.012 & 0.217 & -0.058 & 0.214 & 87 \\
Walter & -0.096 & 0.214 & 0.000 & 0.265 & 22 \\
\hline
\end{tabular}

measurements of the plates with GRS was calculated by the following conditional equations

$$
\begin{aligned}
& X=a x+b y+c+l x^{2}+m x y+n D x, \\
& Y=d x+e y+f+p x y+q y^{2}+r D y,
\end{aligned}
$$

where $x, y$ are the measured coordinates, $X, Y-$ the standard coordinates, $D$ - the diameters of the images of stars, $a, b, c, \ldots, r-$ the plate constants. The terms with diameters take into account the influence of the optical aberration of the astrograph - coma.

The positions of 113 objects were determined from 2-10 plates in the system PPM reference catalogue (Rizvanov \& Dautov 1998). Formal standard errors of reduction of the measurements made by conditional equations vary between $0.15^{\prime \prime}$ and $0.4^{\prime \prime}$ on both coordinates. Formal standard errors of median values of $\alpha, \delta$ of GRS, obtained from 2-10 plates, are within the limits of $0.25^{\prime \prime}-0.05^{\prime \prime}$.

\section{Estimation of accuracy of the observed positions of 113 GRS}

To make an estimate of the accuracy of the observations we compared them with the data of the Hipparcos catalogue and the article of Walter et al. (1991). For each of the comparisons of GRS the positional differences $(\mathrm{O}-\mathrm{C})$ :

$$
\begin{aligned}
\Delta \alpha \cos \delta & =\left(\alpha_{\mathrm{GRS}}-\alpha_{\mathrm{CAT}}\right) \cos \delta_{\mathrm{CAT}}, \\
\Delta \delta & =\left(\delta_{\mathrm{GRS}}-\delta_{\mathrm{CAT}}\right),
\end{aligned}
$$

were determined and analysed where (CAT) is the Hipparcos or Walter position. The differences between GRS and Hipparcos or Walter have been determined at the epoch of the observation of GRS by computing Hipparcos and Walter positions at this epoch with the Hipparcos and Walter proper motions. Results of the comparison with the Hipparcos catalogue are shown in Table 1 and as a function of the magnitude in Fig. 1.

87 objects out of 113 GRS appeared in the Hipparcos catalogue. The list of Walter et al. (1991) contains 52 GRS in the optical region and 23 GRS in the radio window. The $\Delta \alpha \cos \delta$ reaches high values in the optical region (GRS - Walter). Among the 52 GRS, the 13 objects have $(\mathrm{O}-\mathrm{C}) \gg 1^{\prime \prime}$. The optical coordinates of this list are probably out of date and need to be improved.
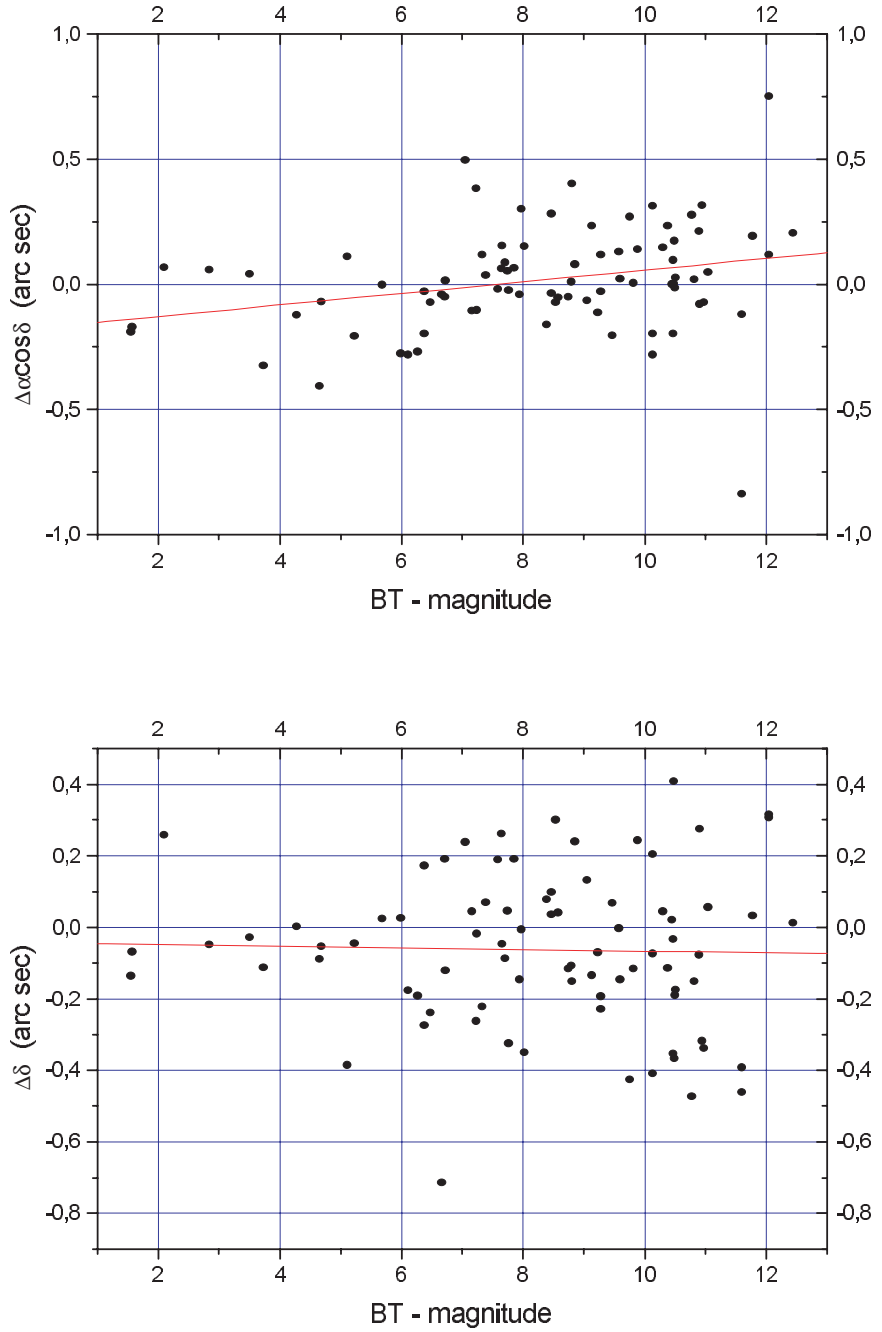

Fig. 1. Differences in right ascension and declination between GRS and Hipparcos catalogue at epoch of observation of GRS as a function of the magnitude. Differences are in the sense GRS - Hipparcos.

In the radio window, $(\mathrm{O}-\mathrm{C}) \gg 1^{\prime \prime}(\mathrm{GRS}$ No. 113 with a large proper motion). The results of the comparison with the Hipparcos catalogue are $\Delta \alpha \cos \delta=-0.097^{\prime \prime}$, $\Delta \delta=-0.109^{\prime \prime}$. Therefore, upon further consideration, this $(\mathrm{O}-\mathrm{C})$ was not included. For the rest, the $(\mathrm{O}-\mathrm{C})<1^{\prime \prime}$ : $8(\mathrm{O}-\mathrm{C})<0.1^{\prime \prime}$, which is $36 \%$ of the total quantity; $10(\mathrm{O}-\mathrm{C})<0.2^{\prime \prime}$, corresponding to $43 \% ; 14(\mathrm{O}-\mathrm{C})<0.3^{\prime \prime}$, corresponds to $61 \%$.

The median values of differences of coordinates between the GRS and Hipparcos, Walter (in the radio window) and its formal standard error are tabulated in Table 1 . The table successively indicates: the catalogues of comparison; mean values of $\Delta \alpha \cos \delta ; \sigma_{\alpha}$ - its formal standard error; mean values of $\Delta \delta ; \sigma_{\delta}$ - its formal standard error; number of the common stars.

Table 2 shows observed coordinates of 113 GRS. The table indicates successively: the serial number of GRS; the Hipparcos number; right ascension and declination of the GRS equinox J.2000.0 and the epoch of the 
observations; BT - blue magnitude from Hipparcos; number of plates; the epoch of the observation (year minus 1900).

The data shown in Fig. 1 can be considered as containing systematic errors of type $\Delta \alpha_{\mathrm{m}}, \Delta \delta_{\mathrm{m}}$.

\section{Conclusion}

1. The coordinates of 113 GRS are close to the FK5 system.

2. The observed positions of GRS have an accuracy which is consistent with the accuracy of modern photographic positional observations.

Acknowledgements. This work was supported by the Competitive Center of Fundamental Natural Sciences at St.-Peterburg State University.

\section{References}

Ma, C., \& Feissel, M. 1997, IERS TECHNICAL NOTE 23

Kolchinsky, I. G., \& Onegina, A. B. 1979, Astrometrija and Astrophysic 39, 57 (in rus.)

Perryman, M. A. C., et al. 1997, ESA, The Hipparcos and Tycho Catalogues, SP 1200, 1-17

Polojentsev, D. D., \& Potter, Ch. I. 1979, Astrometrija and Astrophysica, 39, 63 (in rus.)

Rizvanov, N. G., \& Kitkin, V. N. 1993, Kinematics and physics celestial bodies, 3, 75 (in rus.)

Rizvanov, N. G., \& Dautov, I. A. 1998, Kinematics and physics celestial bodies, 5,457 (in rus.)

Rizvanov, N. G., Dautov, I. A., \& Kibardina, M. I. 1998, The optical coordinates of 113 Galactic radio sources, Kazan, Dep. VINITI 08.09.98, No 861-B98, 11 (in rus.)

Walter, H. G., Hering, R., \& de Vegt, Ch. 1991, A\&AS, 86, 357 